\title{
Germanica
}

\section{Hermann Broch über die Figur des Siegers und die Frage der „demokratischen Propaganda“}

Stephanie BAUMANN : Hermann Broch, la figure du vainqueur et la

"propagande démocratique»

Stephanie BAUMANN : Hermann Broch about the figure of the winner and

'democratic propaganda'

\section{Stephanie Baumann}

\section{OpenEdition}

Journals

\section{Édition électronique}

URL : https://journals.openedition.org/germanica/8593

DOI : 10.4000/germanica.8593

ISSN : 2107-0784

\section{Éditeur}

Université de Lille

\section{Édition imprimée}

Date de publication : 1 juin 2020

Pagination : 149-166

ISBN : 978-2-913857-45-2

ISSN : 0984-2632

\section{Référence électronique}

Stephanie Baumann, „Hermann Broch über die Figur des Siegers und die Frage der "demokratischen Propaganda"', Germanica [Online], 66 | 2ème trimestre 2020, Online erschienen am: 02 Januar 2022, abgerufen am 08 Januar 2022. URL: http://journals.openedition.org/germanica/8593 ; DOI: https:// doi.org/10.4000/germanica.8593 


\title{
Hermann Broch über die Figur des Siegers und die Frage der „demokratischen Propaganda“
}

\author{
Stephanie Baumann \\ Université Polytechnique Hauts-de-France
}

Ob Hermann Broch und Siegfried Kracauer einander begegnet sind, ist unklar - jedenfalls finden sich in Kracauers Nachlass keine Spuren eines Dialogs. Sie hatten jedoch gemeinsame Bekannte: Erwin Panofsky, Ernst Bloch und Hannah Arendt, die mit Broch befreundet war und die Kracauer im Pariser Exil traf. Außerdem standen beide zwischen 1945 und 1946 bzw. 1948 mit dem Propaganda-Spezialisten und Sozialpsychologen Hadley Cantril in Kontakt, der am Radio Research Project von Paul Lazarsfeld mitarbeitete ${ }^{1}$. Broch und Kracauer bewegten sich in New York im Umfeld der New School of Social Research und bezogen Stipendien von der Bollingen Foundation, Broch für Massenwahntheorie und Kracauer für Theory of Film ${ }^{2}$. Die fehlenden

1. - Vgl. die Korrespondenzen mit Hadley Cantril im Kracauer- bzw. BrochNachlass im Deutschen Literaturarchiv Marbach. Broch bezog zwischen 1942 und 1944 ein Rockefeller-Stipendium, das über Cantrils Institut lief (Cantril war Leiter des Office of Public Opinion Research an der Princeton University). Vgl. Paul Michael Lützeler, „Genese eines Exilprojekts: Hermann Brochs Entwürfe zur ,Massenwahntheorie"“, Weimarer Beiträge 60 (2014) 2, S. 216-233, hier S. 223f. Paul Michael Lützeler sei für zahlreiche Hinweise herzlich gedankt.

2. - Beide unterzeichneten einen offenen Brief (in Saturday Review of Literature, Jg. 32, Nr. 36 vom 3.9.1949, S. 26), der die Bollingen Foundation gegen den Vorwurf faschistischer Tendenzen verteidigte, die sie sich wegen der Verleihung eines Preises an 
Spuren eines Austauschs trotz räumlicher Nähe sind symptomatisch für die Unterschiedlichkeit ihrer Totalitarismus-Analysen. In Brochs Schriften sind die Grenzen zwischen diskursivem und literarischem Schreiben stets porös ${ }^{3}$. Er verbindet Überlegungen geschichtstheoretischer, erkenntnistheoretischer, psychologischer und anthropologischer Art, um Ansätze einer politischen Theorie zu entwickeln. Trotz der fundamentalen Unterschiedlichkeit gibt es Berührungspunkte mit Kracauer. So verfasste Broch einen Text über den Zeitgeist von 1940, der von einem Film, nämlich Gone with the Wind, Ausgang nimmt. Auch taucht Kracauers Essay „Das Ornament der Masse“ (1927) in der Bibliographie seiner Fragment gebliebenen Schrift auf, an der er von 1939 bis 1948 im amerikanischen Exil arbeitete ${ }^{4}$. Wie andere Forschungsprojekte, die von Emigranten in den USA durchgeführt wurden (etwa das der exilierten Frankfurter Schule über die autoritäre Persönlichkeit), wollte Broch mit seiner Studie einen intellektuellen Beitrag zur Bekämpfung des Faschismus leisten. Er beschränkt sich in der Schrift, zu der ihn Gespräche mit Elias Canetti animierten ${ }^{5}$, nicht auf die Gegenwartsanalyse, sondern stellt auch Überlegungen hinsichtlich der Möglichkeit einer demokratischen „Gegenbekehrung“ an. Seine Reflexionen gründen auf einer vom Neukantianismus und Heinrich Rickert beeinflussten und in Auseinandersetzung mit Max Scheler formulierten Werttheorie ${ }^{6}$. Broch skizziert sie bereits im dritten Teil Hugenau oder die Sachlichkeit 1918 (1932) seiner Romantriologie Die Schlafwandler. Er verknüpft sie mit einer Stil-Theorie des Ornaments, ein Motiv, das seine Aufmerksamkeit möglicherweise auf Kracauers Essay gelenkt haben mag.

Ausgehend von dem (gegen Spengler gerichteten ${ }^{7}$ ) Wertzerfallessay werde ich zunächst die Bedeutung der Werttheorie für Brochs Totalitarismus-Analyse erläutern. Während Kracauer in Totalitäre Propaganda - ähnlich wie Arendt - von der propagandistischen

Ezra Pound zugezogen hatte. Olivier Agard, „,De Caligari à Ratti: Philosophie de la culture et lecture du fascisme chez Hermann Broch et Siegfried Kracauer", Austriaca. Cahiers universitaires d'information sur l'Autriche, Dez. 2002, Nr. 55, S. 129-155, hier S. 129.

3. - Vgl. dazu Hannah Arendt, „Einleitung zu den Essay-Bänden von Hermann Broch. ,Der Dichter wider Willen“" [1955], in: Dies./Hermann Broch, Briefwechsel 1946 bis 1951, Hrsg. v. Paul Michael Lützeler, Frankfurt a.M., Jüdischer Verlag, 1996, S. 185-226, bes. S. 186.

4. - Hermann Broch, Massenwahntheorie. Beiträge zu einer Psychologie der Politik, KW 12, Frankfurt a.M., Suhrkamp, 1979.

5. - Paul Michael Lützeler, Hermann Brochs Kosmopolitismus: Europa, Menschenrechte, Universität, Wiener Vorlesungen, Wien, Picus, 2002, S. 24.

6. - Vgl. Endre Kiss, „Die Auseinandersetzung mit Max Scheler“, in: Paul Michael Lützeler/Michael Kessler (Hrsg.), Brochs theoretisches Werk, Frankfurt a.M., Suhrkamp, 1988, S. 109-121.

7. — P. M. Lützeler, Brochs Kosmopolitismus, a.a.O., S. 27. 
Konstruktion einer Scheinrealität spricht, die im Nihilismus wurzelt, sieht Broch in Massenwahntheorie eine faschistische Scheinethik am Werk, welche auf der Figur des ,Siegers“ gründet, einer Figur, die schon zu Weimarer Zeiten im Kino, aber auch im Sport zunehmend begeisterteren Zuspruch findet. Fordert Broch in den Jahren 1939 bis 1941 dazu auf, die Faschismen mit einem kriegerischen Pazifismus zu bekämpfen, geht es für ihn nach dem Krieg darum, den ,mythischen Gehalt" des Sieges als Götzen zu entwerten und die Mängel bisheriger Formen der Demokratie zu beheben.

\section{Ornamentlosigkeit und Wertzerfall}

Wie Kracauer interessierte sich auch Broch für Architektur. Im Roman Hugenau oder die Sachlichkeit, dessen Handlung sich im Kriegsjahr 1918 zuträgt, finden sich im Wertzerfallessay, der den Roman gleichsam girlandenhaft ${ }^{8}$ durchzieht, mehrere Passagen über den Zusammenhang von Architektur, Ornament und Moderne. Die dort angestellten Reflexionen ähneln Kracauers Überlegungen in „Das Ornament der Masse“ (1927) zwar in einigen Punkten, weisen jedoch letztlich in eine andere Richtung. Während Kracauer den Ort einer Epoche im Geschichtsprozess aus der Analyse ihrer ,unscheinbaren Oberflächenäußerungen" ${ }^{\text {" }}$ zu bestimmen sucht, operiert Broch zur Charakterisierung der Epoche mit dem Begriff des Stils. Ähnlich wie Alois Riegl in Stilfragen. Grundlegungen zu einer Geschichte der Ornamentik (1893) ${ }^{10}$ glaubt Broch, dass aus dem Stil einer jeden Zeit ein überindividueller Epochengeist abzulesen wäre: „Stil ist etwas, das alle Lebensäußerungen einer Epoche in gleicher Weise durchzieht" "11. Geht es Kracauer vornehmlich um unbewusste Äußerungen, deren Deutung die Erkenntnis einer Epoche ermöglicht, sind es bei Broch die bildende Kunst und die Architektur, aus denen sich eine solche Erkenntnis direkt gewinnen lässt. Das Ornament, das in der Moderne zur Formel des Raumgedankens, bzw. des Stils selbst wird, enthält ähnlich Kracauers Massenornament die „Formel der ganzen Epoche und ihres Lebens“12.

8. - Vgl. Paul Michael Lützeler, „Die Schlafwandler: Architektur und Ornament", in: Ders., Die Entropie des Menschen. Studien zum Werk Hermann Brochs, Würzburg, Königshausen \& Neumann, 2000, S. 33-44, hier S. 40.

9. - Siegfried Kracauer, „Das Ornament der Masse“, in: Ders., Essays, Feuilletons, Rezensionen 1924-1927, Werke, Bd. 5.2., Hrsg. v. Inka Mülder-Bach, Berlin, Suhrkamp, 2011, S. 612-624, hier S. 612.

10. - Broch besuchte in jungen Jahren eine Vorlesung des Wiener Kunsthistorikers Julius Schlosser, einem Fortsetzer der von Alois Riegl begründeten kunsthistorischen Wiener Schule. Vgl. P. M. Lützeler, „Architektur und Ornament“, a.a.O., S. 34.

11. - Hermann Broch, Die Schlafwandler, KW 1, Frankfurt a.M., Suhrkamp, 1994, S. 444.

12. — Ebd., S. 445. 
Magische Bedeutung hat für Broch die Tatsache, dass die dem Krieg und dem Sterben vollkommen verhaftete Epoche des Ersten Weltkriegs, die er in Hugenau darstellt, ,in einem Stil leben muß, der kein Ornament mehr hervorzubringen mag"13. Sieht Kracauer ein Jahrzehnt später im Massenornament eine neue Form des Ornaments, das die Abstraktheit der Moderne abbildet, interpretiert Broch gerade die Ornamentlosigkeit in der Architektur als Zeichen einer solch übersteigerten Abstraktheit. Die Anti-Ornamentik von Adolf Loos wertet er anders als Kracauer, der auch von sinnlosen oder überflüssigen Ornamenten spricht ${ }^{14}$, polemisch als Ausdruck einer modernen Unfähigkeit, Ornamente zu schaffen ${ }^{15}$. Für ihn erscheint die zeitgenössische Bauweise als Symptom eines funktional ausgerichteten Denkstils, dem er eine aggressive Logik attestiert ${ }^{16}$.

Ein Zusammenhang zwischen Ornamenttheorie und Werttheorie ergibt sich bei Broch, indem er den Stil einer Epoche als Ausdruck ihres sogenannten Wertstrebens betrachtet. Ein Stil verweist seiner Auffassung nach stets auf die Einheit einer Epoche - eine weitere Vorstellung, die Kracauer in seinem Spätwerk History - The Last Things before the Last in Frage stellen wird ${ }^{17}$. Laut Broch repräsentiert der

13. - Ebd.

14. - Bei Kracauer tauchen bereits zwischen 1921 und 1925 mehrfach Bemerkungen über das Ornament in der Architektur auf. Vgl. Siegfried Kracauer, „Der verbotene Blick“ [1925], in: Ders., Essays, Feuilletons, Rezensionen 1924-1927, Berlin, Suhrkamp, 2014, S. 224-227, hier S. 224. In einem Text über eine Werkbundausstellung kommentiert er das "Substraktionsverfahren“ durch den Abzug der überflüssigen Ornamente wie folgt: „Indessen ist durch bloße Negation das Positive noch längst nicht erlangt“. Siegfried Kracauer, „Das Suchen nach Form“ [1925], in: Ebd., S. 223224, hier S. 223.

15. - Hermann Broch, „Notizen zu einer systematischen Ästhetik“ [1912], in: Ders., Schriften zur Literatur 2, KW 9/2, S. 11-35, hier S. 22. In einem anderen Punkt ähneln sich Brochs und Kracauers Auffassungen. Beide werten den Film als Träger eines neuen Epochenstils. Im Nachlass von Broch finden sich Entwürfe zu Drehbüchern - etwa eine Adaptation seiner Novelle Ophélia [1920] und des Romans Die Unbekannte Größe [Das Unbekannte X, 1935]. 1946 plant er mit Friedrich Torberg eine Adaptation von Werfels Roman Stern der Ungeborenen. Bei Brochs Drehbüchern handelt es sich um Literaturverfilmungen, obwohl er - wie Kracauer - literarisierte Filme eigentlich ablehnt. Vgl. Hermann Broch an Rudolf Brunngraber, 2.12.1948, in: Ders., Briefe 3 (1945-1951), KW 13/3, S. 277. Jürgen Heizmann, „Massenmedium: Hermann Broch und der Film", in: Alice Staskova/Paul Michael Lützeler (Hrsg.), Hermann Broch und die Künste, Berlin, de Gruyter, 2009, S. 75-92, hier S. 87.

16. - H. Broch, Die Schlafwandler, a.a.O., S. 437 und S. 496. P. M. Lützeler, „Architektur und Ornament“, a.a.O., S. 36.

17. - Kracauer polemisiert in diesem Zusammenhang u.a. gegen Benedetto Croce. Vgl. Siegfried Kracauer, Geschichte - Vor den letzten Dingen, Werke Bd. 4, Hrsg. v. Ingrid Belke unter Mitarbeit v. Sabine Biebl, Frankfurt a.M., Suhrkamp, 2009, S. 76. Vgl. dazu Stephanie Baumann, Im Vorraum der Geschichte. Siegfried Kracauers History - The Last Things before the Last, Paderborn, Konstanz University Press, 2014, insbes. S. 131-160. 
Stil das absolute Wertzentrum einer Kultur, ist sinnlich wahrnehmbarer Zeitgeist. Der „Unstil““ der säkularisierten Moderne verweise mithin auf die Abwesenheit eines idealen Wertzentrums. Die Einheit, die einst durch das religiöse Wertsystem hergestellt wurde, sei in der Moderne einer Pluralität von Partialwertsystemen gewichen, die sich immer weiter ausdifferenzieren:

[...] mitleidlos durchdringt das Abstrakte die Logik jedes einzelnen Wertschaffens, und ihre Inhaltsentblößung [...] radikalisiert auch die einzelnen Wertgebiete so sehr, da $\beta$ diese [...] voneinander sich trennen, sich parallelisieren und, unfähig einen gemeinsamen Wertkörper zu bilden, paritätisch werden, - gleich Fremden stehen sie nebeneinander [...] ein jedes in seiner Autonomie, entfesselt ${ }^{\top 18}$.

Wie kommt es, dass sich die Individuen der zeitgenössischen Kriegsideologie so einfach fügen? - lautet Brochs Leitfrage. Wie ist es möglich, dass es „keinen anficht, wenn Tausende in Stacheldrähten hängen" 19 ? Er erklärt diesen Tatbestand mit der von ihm diagnostizierten Zersplitterung menschlichen Daseins und Erlebens in der Moderne. Ein jeder folge seiner individuell absolut gesetzten Logik: der Militär, der Völker ausrottet, der Wirtschaftsführer, der dem Geschäft unter Vernichtung aller Konkurrenz zum Erfolg verhilft, der Maler, welcher esoterische Gebilde produziert, der politische Mensch, der sein politisches Ziel bis hin zur Diktatur zu verabsolutieren sucht, der bürgerliche Faiseur, der nach Reichtum strebt - ihr aller Handeln werde von auf den eigenen Bereich reduzierten Prinzipien geleitet: „Krieg ist Krieg, l'art pour l'art, in der Politik gibt es keine Bedenken, Geschäft ist Geschäft“. An die Stelle des Menschen als Gottes Ebenbild tritt der sogenannte Berufsmensch ${ }^{20}$. Der Denkstil der Epoche, in dem sich die Partialsysteme Militär, Wirtschaft, Politik gegeneinander durchzusetzen suchen, ist von einer metaphysischen Rücksichtslosigkeit gekennzeichnet, die einzig der jeweiligen Sache dient ${ }^{21}$. Zum Kontrast verweist Broch auf die europäische Vormoderne, die ein ideales Wertzentrum kannte, d.h. den christlichen Glauben als „Plausibilitätspunkt, bei dem jede Fragekette endigte“22.

Die Sehnsucht nach einer Führer-Figur entspringt nach Brochs Deutung aus dem Bedürfnis, das als wahnsinnig wahrgenommene und dennoch passiv akzeptierte politische Geschehen mit Motivation $\mathrm{zu}$ versehen ${ }^{23}$. Motivationsstiftend könnte ein Religionsstifter wie

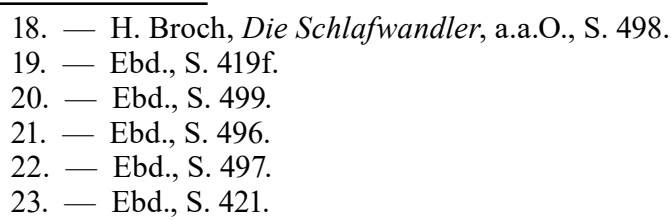


auch ein Demagoge wirken. Ersterer unterschiede sich von letzteren durch sein Ethos. Broch gebraucht in diesem Zusammenhang auch den Begriff des echten Heilsbringers ${ }^{24}$ - eine Abwandlung der alttestamentarischen Figur des echten (bzw. falschen) Propheten -, dessen Echtheit sich laut Broch darin manifestiert, dass er sich auch im Kollektiv stets an das Individuum richtet. Der Demagoge hingegen versucht über die Mobilisierung der Emotionen des Individuums dessen Aufgehen in der Menge zu bewirken, um den eigenen Machtanspruch durchzusetzen: Er ,setzt seine eigene irdische Person zum Symbol für die Gemeinschaft, die er repräsentieren will und für deren ,Sieg'““25, wobei Sieg Macht und Herrschaft symbolisiert. Genau dies geschieht laut Broch im Faschismus, der seine Attraktivität daraus bezieht, dass er die erwähnten Teilwertsysteme durch eine falsche Totalität ersetzt.

\section{Faschismen als Zerrbilder echter Werttotalität}

Anders als Elias Canetti, der in Masse und Macht (1960) unterschiedlich zu bewertende, auch positive, durch libidinöse Bindungen zusammengehaltene Massenerscheinungen beschreibt (etwa die Festmasse), konzentriert sich Brochs Interesse auf die faschistische Massenbildung, die er zu bekämpfen sucht ${ }^{26}$. Er interessiert sich wie Freud vornehmlich für das Individuum und für das Pathologische des Phänomens der „Masse“, das er 1939 im Rahmen eines interdisziplinären Forschungsinstituts über politische Psychologie aus empirischnaturwissenschaftlicher, historisch-theoretischer und politisch-pädagogischer Perspektive erforscht wissen wollte ${ }^{27}$. Albert Einstein, mit dem er sich über das Projekt austauschte, vermittelte ihn an den Psychologen und Propaganda-Spezialisten Hadley Cantril, der 1940 an der Princeton University das Office of Public Opinion Research gründete ${ }^{28}$.

24. - H. Broch, Massenwahntheorie, a.a.O., S. 300.

25. - Ebd., S. 301.

26. - Bei Broch ist das Phänomen von Anbeginn negativ besetzt. In einem offenen Brief an Franz Blei beschreibt er die Menschenmenge, in der er sich verlor, als 1918 die Deutschösterreichische Republik ausgerufen wurde. Er spricht von einer Sogwirkung und einem Erlebnis, das er als ,psychotisch“ einstuft. Vgl. An Franz Blei, [Offener Brief, Wien, Dezember 1918], Die Straße, in: Briefe 1 (1913-1938), Frankfurt a.M., Suhrkamp, 1986, S. 30-35, hier S. 30 und 32. Vgl. dazu Dagmar Barnouw, „Abwehr und Ambivalenz. Zum Konzept der Masse bei Hermann Broch und Alfred Döblin“, in: Michael Kessler/Paul Michael Lützeler (Hrsg.), Hermann Broch. Das dichterische Werk. Neue Interpretationen, Tübingen, Stauffenburg Colloquium, 1987, S. 221-229.

27. - Lützeler unterscheidet drei Entstehungsphasen der Schrift zwischen 1939 und 1943-48 mit jeweils unterschiedlichen Zielsetzungen. Vgl. Paul Michael Lützeler, „Genese eines Exilprojekts...“, a.a.O., S. 218.

28. - Ebd., S. 223. Cantril war der Verfasser des Buches The Invasion from Mars, in dem es um die Massenpanik ging, die Orson Welles' Radiosendung nach $\mathrm{H}$. G. Wells' Roman War of the Worlds ausgelöst hatte. 
Broch verbindet in Massenwahntheorie diese Reflexionen mit Überlegungen zum Geschichtsprozess. Er sucht historische Gesetze des Phänomens zu beschreiben, die er wie schon seine Ornamenttheorie wiederum zu einer Werttheorie in Beziehung setzt. Grundsätzlich zeichne sich der historische Verlauf durch psychische Zyklen aus, innerhalb derer „Zeiten der Vernunft“ und ,Zeiten des Wahns“ einander abwechseln ${ }^{29}$. Diese können im Übrigen dazu beitragen, die Wellenbewegung ökonomischer Zyklen zu erklären, die laut Broch nicht allein über die „Mechanik der Kapitalkonzentration“ zu begreifen sind (er nennt als Beispiel Phänomene der Börsenspekulation) ${ }^{30}$. Brochs Beschreibung des Gesetzes psychischer Zyklen erinnert im Übrigen stark an Spenglers naturhafte Geschichtskonzeption, zu der er sich gleichwohl wiederholt negativ äußert und deren politische Implikationen er ablehnt ${ }^{31}$. Der Phase des Kulturaufbaus unter der Herrschaft eines zentralen Wertes folgt laut Broch eine Phase des „Hypertrophiewahns“, wenn dieses System an seine „Unendlichkeitsgrenzen“ gelangt. Daraufhin kommt es zu einer „Wiederetablierung der Realität" und zu einem Übergang in die Phase der Wertzersplitterung, welche von einem "Zerrissenheitswahn“ begleitet wird. Schließlich werde nach einem neuen zentralen Wert gesucht ${ }^{32}$. Broch baut seine Argumentation also nicht auf der historisch-gesellschaftlichen Dimension des Geschehens auf, sondern argumentiert mit pathologisierenden psychologischen Begriffen. Der Wertzerfall in der modernen Gesellschaft und das damit verbundene Abhandenkommen der seelischen Sicherheit (laut Broch ein vornehmlich religiöses Bedürfnis ${ }^{33}$ ) begünstige beim Individuum das Aufkommen von Panik. Diese zeitige immer dann massenpsychologische Wirkung, wenn das Individuum den Wertverlust im Kollektiv zu kompensieren versuche. Die Panik entlade sich dann vornehmlich in Gewalt gegen das Non-Ich, d.h. gegen alles Fremde ${ }^{34}$. Führer-Figuren könnten ein Kollektiv sowohl auf den Weg der sogenannten Irrationalbereicherung

29. - H. Broch, Massenwahntheorie, a.a.O., S. 292-295.

30. - Ebd., S. 291.

31. - Vgl. Paul Michael Lützeler, „Europäischer Kulturzerfall. Brochs ,Schlafwandler' und Spenglers ,Untergang des Abendlandes““, in: Ders., Europäische Identität und Multikultur. Fallstudien zur deutschsprachigen Literatur seit der Romantik, Tübingen, Stauffenberg Verlag, 1997, S. 87-105, hier S. 93, S. 95. In Massenwahntheorie wirft Broch Spengler vor, die Parallelität der Stil-Abläufe in unterschiedlichen Kulturen „zu Tode zu hetzen“. Vgl. H. Broch, Massenwahntheorie, a.a.O., S. 184.

32. - Ebd., S. 292.

33. - Hermann Broch, „Zur Diktatur der Humanität innerhalb einer totalen Demokratie“ [1939], in : Politische Schriften, KW 11, Frankfurt a.M., Suhrkamp, 1978, S. 24-71, hier S. 61 .

34. - Monika Ritzer, „Massenwahntheorie“, in: Michael Kessler/Paul Michael Lützeler (Hrsg.), Hermann Broch Handbuch, Berlin/Boston, De Gruyter, 2016, S. 433460, hier S. 443. 
wie auch auf den Weg des Rationalverlustes und der archaisch-infantilen Triebauslebung bringen, vor allem über Prozesse der Personifikation und Identifikation ${ }^{35}$. Die Faschismen hätten diese Mechanismen genial erkannt und zur Bildung einer politischen Überzeugung mobilisiert, die nur auf Sieg ausgerichtet ist, laut Broch dem wirksamsten „Ansteckungsträger" faschistischer Überzeugungen ${ }^{36}$. Dieser erfülle die Funktion eines ,Zerrbild[s] echter Werttotalität“"37.

Über die NS-Propaganda äußert sich Broch 1939 in einem Buchentwurf „Zur Diktatur der Humanität innerhalb einer totalen Demokratie", den er an Freunde (darunter Stefan Zweig) verschickte. In der Vorbemerkung kündigt er an, dass er sich von seiner historischen Erfahrung ausgehend mit der „Bildwirkung als Massenführung“ befassen wolle ${ }^{38}$. Broch unterscheidet zwei Angriffspunkte deutscher Propaganda: die Angst der herrschenden Klasse vor dem Bolschewismus und die Tendenz der Untermittelklasse wie der Mittelklasse, ,sich einer Moral des ,Warum-nicht“ anzuschließen“39. Die falsche Werttotalität der Nazis entspräche der Kombination aus einer konservativen Übermoral und einer revolutionären Untermoral ${ }^{40}$, , ,[...] m.a.W. es wird jedem recht getan, wie es zum Wesen eines jeden guten Inseratengeschäfts gehört" ${ }^{\text {‘41 }}$. Letztere sei die eigentliche Weltanschauung moderner Diktaturen, der Bruch mit allen Prinzipien der Humanität, mit

[...] der absoluten Ethik an sich, freilich dadurch gemildert, daß die Lizensierung der Frage ,Warum nicht morden?', Warum nicht rauben?' nur in Ansehung einer Minoritätengruppe, den Juden, statthaben soll, eine Einschränkung, welche die weltanschauliche Gefahr der Moralaufhebung für die herrschenden Klassen in einer durchaus angenehmen Weise camoufliert ${ }^{42}$.

Broch bringt diesen Vorgang mit einer Stimmung von geisterhafter Unwirklichkeit in der Großstadt in Zusammenhang, in der das Lebensgefühl von dem Bewusstsein um die „Unbewältigbarkeit einer einstmals bewältigbaren Welt" dominiert sei ${ }^{43}$. Mit einer zeittypischen satanologischen Metaphorik spricht er davon, im unkontrollierbar gewordenen Weltgeschehen werde nur noch dem „Teufelsgott“

35. - H. Broch, „Zur Diktatur der Humanität“, KW 11, a.a.O., S. 55.

36. - H. Broch, Massenwahntheorie, a.a.O., S. 354, S. 310.

37. - Ebd., S. 372.

38. - Ebd., S. 24.

39. - H. Broch, „Zur Diktatur der Humanität“, a.a.O., S. 31.

40. - Ebd.

41. - Ebd.

42. - Ebd.

43. - Ebd., S. 49. 
Konjunktur gehuldigt ${ }^{44}$, während sich unter den Menschen die apokalyptische Ahnung breit gemacht habe, dass eine Wiederkehr einstiger prosperity nicht zu erwarten stehe ${ }^{45}$. Dem entspricht ein Wandel im Bereich des politischen Imaginären: während sich die Vorstellungswelt des Menschen in der Ur-Demokratie mit den Belangen selbiger gedeckt habe, entspringe die Vorstellungswelt des zeitgenössischen Menschen nicht dem Gemeinwesen und auch nicht der Geldsucht, sondern der Kinoindustrie und den Illustrierten ${ }^{46}$ : „er flüchtet mit seiner Seele ins Dämonische und mit seinem Leib ins Kino, wo dem Kinohelden zuverlässig die Bewältigung des Unbewältigbaren im allerbekanntesten Wunschtraum gelingt" 47 . Nun nimmt der Kinoheld als Identifikationsfigur die Stelle Gottes ein, um das Erlösungsbedürfnis des modernen Menschen zu befriedigen. In revolutionären wie antirevolutionären, in kriegerischen wie pazifistischen Filmen wird die Guillotine zum ,handgreifliche[n] Opferinstrument“48.

Neu ist für Broch die Industrialisierung des Dämonischen im Prozess der Identifikation mit einer Siegerfigur, wobei wie bei Kracauer der Film und der Sport potenziell dieselbe Funktion erfüllen können. Die Begeisterung für sportliche Rekorde erscheint bei Broch als Suche nach einer fiktiven Wertspitze, die Identifikation mit dem Sieger als ,eigentliche Denkform der modernen Massenseele“49. Dieselbe Beobachtung formuliert Kracauer, wenn auch anders, ironisch getönt, in dem Essay „Sie sporten“ aus dem Jahr 1927:

[...] Die Sportgeistigen beseelt der Wille zum Sieg. Da sie nicht wissen, was sie besiegen sollen, suchen sie Rekorde zu brechen. Auch die unterlegene Mannschaft hat sich wacker gehalten, aber der gebrochene Rekord ist so traurig. Immer möchten sie siegen, der Wettkampf stählt den Charakter ${ }^{50}$.

Der moderne Mensch flüchtet aus dem Alptraum in die Zahlenbetäubung, die Broch mit dem Scheitern der Demokratie in Zusammenhang bringt. „Welches Interesse an der Demokratie kann man von einem Träumenden erwarten, außer [das an] den zahlenmäßig sportlichen Wahlresultaten“"51?

44. - So ist etwa die Rede vom Riesendämon Sieg, vgl. H. Broch, Massenwahntheorie, a.a.O., S. 388.

45. - H. Broch, „Zur Diktatur der Humanität“, a.a.O., S. 51.

46. - Ebd., S. 52.

47. - Ebd., S. 53.

48. - Ebd., S. 53f.

49. - Ebd., S. 54.

50. - Siegfried Kracauer, „Sie sporten“ [1927], in: Ders., Essays, Bd. 5.2, a.a.O., S. 524-529, hier S. 525.

51. - H. Broch, „Zur Diktatur der Humanität“, a.a.O., S. 54. Auch Arendt unterstreicht die Bedeutung des Sieges als Wertideal, dessen propagandistische Wirkung sie 
In welche Richtung dieser Zeitgeist treibt, liest er am zeitgenössischen Kino ab: In Gone with the Wind (mit Clark Gable und Vivien Leigh, nach einem Roman von Margret Mitchell) aus dem Jahr 1940 erscheint ihm dieser von der Idee einer neuen Sklaverei durchdrungen, die hier unbewusst von seinen Produzenten ins Bild gesetzt worden sei. Tatsächlich handelte es sich um einen von Hitlers Lieblingsfilmen. Broch sieht in ihm das Hitler'sche Schreckensbild auf die Leinwand gebracht, das Bild des „edle[n], weiße[n] Vaterlandsverteidigers, in den Straßengraben gejagt, auf daß er daselbst verhungere". Der Film, so Broch weiter, handele mithin nicht von der historischen Sklaverei im Süden Amerikas, jener „lieblich-schwerelosen Welt", die dem naiv ,versklavungssehnsüchtigen Auge“" vorgezaubert wird. Es gehe vielmehr um jene Versklavung, die in den Konzentrationslagern durchgesetzt wird ${ }^{52}$. Eine Filmkritik von Kracauer existiert zu diesem Film leider nicht ${ }^{53}$.

\section{„Demokratische Propaganda“?}

Broch verweist wie Kracauer oder auch Arendt auf das Scheinhafte der NS-Inszenierungen. Während letztere jedoch von der Konstruktion einer Scheinrealität durch die NS-Propaganda sprechen, und sich Kracauer für die ästhetische Dimension interessiert, fokussiert Broch auf die Wertdimension des Geschehens, auf welche „demokratische Propaganda“ Einfluss zu nehmen vermag. Es gehe laut Broch darum, die Bevölkerungen aus den geschlossenen Systemen der faschistischen Scheinethik mit ihrer Machtmoral in das „offene Wertsystem der Humanität“ zurückzuführen ${ }^{54}$. Er spricht von einer Propagandakonkurrenz, die gegen den Faschismus aufzubauen sei ${ }^{55}$ und deutet die diktatorialen Gebilde als direkte Abkömmlinge der Demokratien ${ }^{56}$ : die Faschismen hätten richtig erkannt, dass die demokratische Denkform zum europäischen

\footnotetext{
jedoch in der Aufhebung der Klassengegensätze in der totalitären Masse begründet sieht: während es Klassen auf die Durchsetzung von Interessen ankomme, gehe es totalitären Massen um Sieg und Erfolg schlechthin. Vgl. Hannah Arendt, Elemente und Ursprünge totaler Herrschaft. Antisemitismus, Imperialismus, totale Herrschaft, München/Zürich, Piper, 2001, S. 743.

52. - Hermann Broch, „'Gone with the Wind' und die Wiedereinführung der Sklaverei in Amerika“, in: Ders., Schriften zur Literatur 2, Theorie, KW 9/2, Frankfurt a.M., Suhrkamp, 1975, S. 237-246, hier S. 241f. und S. 245.

53. - Er erwähnt ihn lediglich in einer Fußnote. Siegfried Kracauer, „Über den Filmschauspieler [I.]“ [1940], in: Ders., Kleine Schriften zum Film 1932-1961, Werke, Bd. 6.3, Hrsg. v. Inka Mülder-Bach, unter Mitarbeit v. Mirjam Wenzel und Sabine Biebl, Frankfurt a.M., Suhrkamp, 2004, S. 317-320, hier S. 320.

54. - Ebd., S. 63.

55. - Ebd., S. 64.

56. - Hermann Broch, „Theorie der Demokratie 1938-1939“ [1941], in: Ders., Politische Schriften, KW 11, a.a.O., S. 72-80, hier S. 72.
} 
Traditionsbestand gehöre, weshalb sie ihre Staatsform als eigentlich demokratische ausgeben. Dies zeige sich etwa an Volksbefragungen, die als Propaganda-Instrument fungieren, wobei sie demokratische Werte zum reinen Dekorum degradieren ${ }^{57}$.

Broch räumt (anders als Kracauer) der Frage nach der Rolle eines Feindbildes in der NS-Propaganda breiten Raum ein, der Bedeutung des Antisemitismus, der die Verschmelzung der Individuen zur „Masse“ und der „Masse“ mit dem Führer ermöglicht ${ }^{58}$. Die Mobilisierung der faschistischen Masse basiere auf dem Lynchakt als ,ein[em] von der jeweiligen Werttheologie legitimierte[m] Opferakt, [dem] Opferakt eines Irren“59, dessen Funktion darin besteht, Panik abzureagieren. Broch kritisiert in diesem Zusammenhang die Hypertrophie einer Theologie, die daran festhalte, „daß das Menschenopfer notwendig und gottwohlgefällig ist“. Im Passus über die „Phänomenologie des Verfolgten“ reflektiert er über die Figur des Opfers im Christentum. Das Judentum habe sich - nachdem Gott Abraham erlaubte, den Widder und nicht seinen Sohn Isaak zu opfern - nicht so sehr über Gebote, als über Verbote konstituiert, insbesondere über das Verbot, dass man sich mit ,,irgendeiner Art von Blutvergießen [...] Gott wohlgefällig erweisen könnte" 60 . Durch dieses Verbot unterschied sich das Judentum von dem mit dem Menschenopfer identifizierten Heidnischen. Die Figur des Christus hingegen und der Akt der Selbstaufopferung habe sich womöglich zur Wiedereinführung heidnischer Elemente geeignet und - aus Sicht des Judentums und seiner abstrakten Humanitätsauffassung - zu einem Rückschritt geführt, ,zu einer blasphemischen Verzögerung auf dem Wege, der zu einer von jedem Menschenopfer gereinigten Welt führen soll“61.

Broch spricht von einer Propaganda der Tat, die Nazi-Deutschland vermittels „Einpumpung“ jüdischer Flüchtlinge in die demokratischen Länder organisiert habe. Immer wieder finden sich in Massenwahntheorie Formulierungen, die beinahe auf eine Identifikation mit der Sprache des Aggressors hindeuten, was Broch bisweilen bewusst wird ${ }^{62}$. Zur Frage nach den Möglichkeiten einer Bekämpfung des Antisemitismus äußert er sich indessen kaum. Er unterscheidet lediglich eine rationale und eine emotionale Dimension, deren beider Bestimmung jedoch vage bleibt: Auf rationaler Ebene gehe es darum, eine „Stimmung“ zu schaffen, die dem Rassenhass entgegengesetzt sei, bis dieser von selbst aufgegeben werde 63 . Broch formuliert überdies einen in der

57. - H. Broch, Massenwahntheorie, a.a.O., S. 381.

58. - Ebd., S. 309.

59. - Ebd., S. 392.

60. - Ebd., S. 396.

61. - Ebd., S. 397.

62. - Ebd., S. 393 und S. 400.

63. — Ebd., S. 406. 
Diskussion über Propaganda gängigen Einwand gegen alle Formen rationaler Auseinandersetzung mit deren Inhalten: Eine Propaganda des Arguments werte den politischen Gegner auf, anstatt dessen Propaganda abzuwerten ${ }^{64}$, denn ,daß der Antisemitismus sich eine deutsche Märchenwelt aufgebaut hat, einen bösartigen Märchenwald, in dem Zionsweise und Ritualmörder spazierengehen", dies sei sogar dem Antisemiten bewusst, der dieses Wissen allerdings verleugnen müsse 65 .

Worin liegt aber für Broch der qualitative Unterschied zwischen demokratischer und faschistischer Propaganda? Er ergibt sich für ihn vornehmlich aus dem angestrebten Zentralwert. Propaganda könne prinzipiell dazu genutzt werden, wahnhafte Aggressionen zu entfesseln ${ }^{66}$, wie auch demokratische Bekehrung. Eine solche hätte von einer säkularen, wissenschaftlichen und psychologischen Basis ausgehend nach dem Modell der christlichen Mission zu funktionieren ${ }^{67}$. Broch unterscheidet den Begriff der Bekehrung deutlich von dem der Erziehung, wenn er betont, dass erstere nicht mit didaktischen Maßnahmen zu verwechseln sei68. Wie faschistische Propaganda habe auch „demokratische Propaganda" beim Irrationalen anzusetzen. Angstbesänftigung sei nicht in erster Linie durch Aufklärung herbeizuführen, sondern durch Irrationalbereicherung, weil Panik weder durch Abstellung der Panikursachen noch durch Eingehen auf die Forderungen der panikisierten Menge beendet werden könne. Eine solche Bereicherung des Irrationalen herbeizuführen, sei gleichwohl kein einfaches Unterfangen, weil demokratischen Staaten ekstatisierende Elemente wie Sieg und Erfolg fehlten. Es bedürfe stattdessen - Broch bleibt hier sehr vage - „emotionaler Superbefriedigungen“, welche „scharf antisadistisch eingestellt sind, ohne darum selber ins Masochistische umzuschwenken"69. Es gehe um eine feine Austarierung der beiden größten Irrationalitäten, als welche er das Gemeinschaftsgefühl und die freie Persönlichkeitsentfaltung identifiziert. Gemeinschaft ist dabei nicht nur als Multiplikation von Individuen mit einem gemeinsamen Wertsystem zu verstehen. Jede Gemeinschaft setze vielmehr ein Gemeinschaftserlebnis voraus, ein „echtes Werterlebnis“ mit - auf diesem Punkt besteht Broch - ekstasierender Wirkung.

Besonders in diesen Passagen über das Irrationale gerät Brochs Text, wie Daniel Weidner bereits treffend formuliert hat, argumentativ gleichsam ins Schlingern ${ }^{70}$. Wie stellt Broch sich diese ekstasierenden

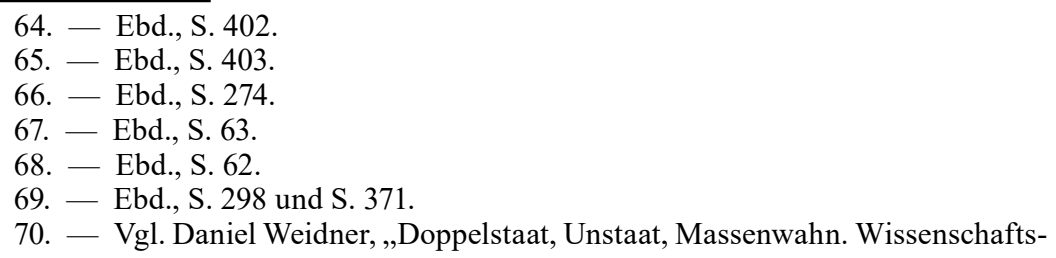

70. - Vgl. Daniel Weidner, „Doppelstaat, Unstaat, Massenwahn. Wissenschafts- 
Erlebnisse konkret vor? Er schreibt, das sogenannte echte Werterlebnis gründe auf einer Ich-Erweiterung, das eine Zweifelsbefreiung auslöst, die das Individuum erlebt, wenn es sein Wertsystem in dem der Gemeinschaft vervielfacht wiederfindet. Dieses Erlebnis könne jedoch, so räumt er ein, auch in einer panikisierten Menge, ja sogar in einer Lynchhorde auftreten ${ }^{71}$. Ob Irrationalwerte in positiver oder negativer Weise wirksam werden, hängt einzig davon $a b$, ob sie das bestehende Rationalsystem intakt lassen oder ob sie es zerstören. Broch stellt sich die Bandbreite zwischen Rationalverlust und Irrationalgewinn auf einer Stufenskala vor. Sie reicht von radikal geschlossenen zu radikal offenen, entwicklungsfähigen Wertsystemen ${ }^{72}$. Um letztere zu fördern, sei die Freiheit des Individuums als Gegengewicht zur Gemeinschaft zu stärken: ,Jeder Kampf gegen Massenwahn muß gewisse Strukturen des Kollektivs auszulöschen trachten - dem Individuum ein Stück innerer Freiheit zurückgeben, allerdings ohne daß hierdurch die ,gesunden ${ }^{6}$ Gemeinschaftsbindungen irgendwie angetastet werden"73.

Wie es der Begriff der Bekehrung nahelegt, beschreibt Broch den Prozess der Rückgängigmachung des Rationalverlusts schließlich nach dem Modell der christlichen Mission. Die demokratische Mission müsse als säkulare Fortsetzung der christlich-katholischen dieselben Phasen wie diese durchlaufen. Ekstatisierende Elemente, die den Rationalverlust bewirkten, würden dabei durch neue Werte ersetzt. Auf eine Phase der Amalgamierung der alten und der neuen Werte hätte eine Phase der Propagandakonkurrenz zu folgen, dann eine der Sicherung des neuen Systems und schließlich die Errichtung eines neuen Tabus ${ }^{74}$. Propaganda interveniert nach diesem Modell in Phase zwei, und Broch formuliert zunächst, welche Art von „Propaganda“ zu vermeiden wäre. Formeln wie Freiheit, Menschenrechte, Menschenwürde seien nicht hinreichend, da sie die Ursachen sozialer und ökonomischer Unsicherheit nicht beheben ${ }^{75}$. Auch eine die Faschismen imitierende Gegenpropaganda sei zu vermeiden, da sie in ihrer Wirkung nicht an das Original herankomme. Sie müsse sich jedoch hinsichtlich ihrer ekstasierenden Wirkung den Faschismen überlegen zeigen ${ }^{76}$. Die Gegenpropaganda der demokratischen Länder bis zum Kriegsausbruch hätte die faschistische Propaganda imitiert, indem sie nicht auf genuin demokratische Werte,

sprache und politisches Denken im Exil“", in: Doerte Bischoff/Christoph Gabriel/Ester Kilchmann (Hrsg.), Sprache(n) im Exil, Exilforschung. Ein internationales Jahrbuch 32/2014, München, edition text + kritik, S. 100-120, hier S. 115.

71. - H. Broch, Massenwahntheorie, a.a.O., S. $276 \mathrm{ff}$.

72. - Ebd., S. $278 \mathrm{ff}$.

73. - Ebd., S. 307.

74. - Ebd., S. 64.

75. - Ebd., S. 352ff.

76. — Ebd., S. 366 und 387. 
sondern auf Gegennationalismus setzte ${ }^{77}$. Damit hätte sie die faschistische Ideologie nicht bekämpft, sondern im Kern bestätigt.

Den emotionalen Stimulanzien der faschistischen Propaganda ist laut Broch gemeinsam, dass sie allesamt auf der Figur des Sieges gründen. Demokratische Bekehrung müsse also die Ebene des Sieges überspringen und auf metaphysischer Ebene ansetzen. Demokratie „hat Platz für Gott“, aber als offenes System ,verträgt sie keine Götzen“78. Glaube kann nicht widerlegt, sondern nur entwertet werden ${ }^{79}$. Die Entwertung des Sieges müsse also zentraler Inhalt demokratischer Propaganda sein: Bekämpfung des Siegesrausches, Ersatz des Siegesjubels durch Siegestrauer. Dies könne nur ein kriegerischer Pazifismus leisten, der den Sieg zum Verbrechen degradiert. Broch verweist hier auf Detektivromane, deren Wirkung nicht zu unterschätzen sei. Er deutet das Genre (ähnlich wie Kracauer in seinem Traktat Der Detektiv-Roman, allerdings mit anderer Stoßrichtung) als Ausdruck von Gewissenskräften, die „Gut von Böse zu unterscheiden wissen und das Böse in der Gestalt des Verbrechers [...] vernichtet haben wollen“. Bekehrender Propaganda gehe es stets um ein Versprechen der Besiegung des Verbrechens. Auf politischer Ebene versuchte Ähnliches erstmalig der Brian-KellogPakt, den 1928 elf Nationen unterschrieben und mit dem sie den Krieg als politisches Mittel als völkerrechtswidrig zu verankern suchten. Brochs Auffassung nach scheiterte der Pakt, weil die Unterzeichner den Pazifismus nicht in die innerstaatliche Gesetzgebung aufnahmen ${ }^{80}$.

\section{„Totale“ Demokratie?}

Entscheidend für die Verteidigung der Demokratie ist die Behebung ihrer Mängel. Broch setzt dazu in den 1940er Jahren ganz auf die normative Macht der Gesetzgebung. Im Zentrum seiner Forderungen steht die Verankerung der demokratischen Prinzipien auf der Ebene des Gesetzes: „die wahre Popularität einer Idee ist, wie das deutsche Propagandaministerium sehr gut weiß, stets im Gerichtssaal verankert; ein wirkungsvolles Gebot hat immer eine Form des Du sollst nicht zu erhalten"81. Antidemokratisches Verhalten hätte mit Strafsanktionen zu rechnen. Eine solche gesetzliche Festschreibung der Demokratie erlaube es nicht nur, den Staat gegen Übergriffe durch seine Bürger zu schützen. Sie erlaubte es auch, durch klare Unterscheidung von Gut und Böse die „Einzelhypertrophierung der verschiedenen autonom gewordenen Unterwertsyteme" zu beseitigen und damit die Unsicherheit als

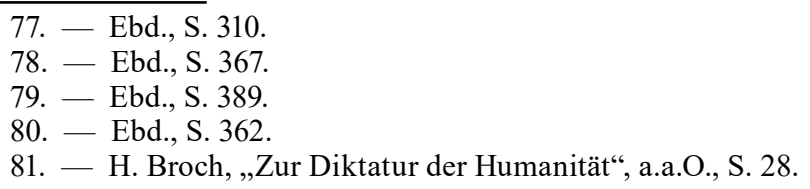


Hauptursache des Zerrissenheitswahns ${ }^{82}$. Eine solche Demokratie wäre als totale zu bezeichnen.

Ist diese Begriffswahl lediglich als theoretische Provokation aufzufassen ${ }^{83}$ ? Daniel Weidner unterstreicht zu Recht, dass Brochs fragmentarisch gebliebener Text als Zeitdokument und Zeugnis eines Verzweifelten gelesen werden muss ${ }^{84}$. An den verschiedenen Textkonvoluten lässt sich zudem eine Entwicklung ablesen. Während Broch Ende der 1930er Jahre betont, dass die Demokratie „Platz für jegliche echte Irrationalbereicherung ${ }^{\text {" } 85} \mathrm{zu}$ schaffen habe, plädiert er ein Jahrzehnt später im letzten Konvolut dafür, dass eine wehrhafte Demokratie eine säkularisierte religiöse Haltung einzunehmen hätte, eine „religiöse Haltung unter Weglassung der religiösen Ideologie“86. Diese Position zeichnet sich in den Fragmenten aus den Jahren 1939 bis 1941 bereits ab. So liest man schon hier an exponierter Stelle - ungeachtet der oben referierten Thesen über die irrationalen Anteile einer demokratischen Propaganda: ,das demokratische Bekehrungswerk ist weniger auf Irrationalbereicherung als auf Verhütung von Rationalverlust ausgerichtet; es ist in erster Linie rationaler Bewußtmachungsprozess" ${ }^{\text {"87. }}$.

Brochs Reaktionen auf den Faschismus erwachsen anders als Kracauers Überlegungen nicht aus einer sozio-politischen Analyse, sondern aus der in den Schlafwandlern formulierten und mehrfach deklinierten These des Wertzerfalls in der Moderne und einem zyklischen geschichtsphilosophischen Modell, das Kracauers Position, die dieser in den 1960er Jahren formuliert, fern steht. Im Gegensatz zu Kracauer konstatiert Broch den Zerfall der einstigen religiösen Werteinheit in der Moderne nicht nur, er bedauert ihn auch. Zwar lässt er die Hoffnung auf einen neuen „Heilsbringer“ im Verlauf seiner Auseinandersetzungen mit totalitärer Politik fallen ${ }^{88}$. Die Hoffnung auf

82. - H. Broch, Massenwahntheorie, a.a.O., S. 362.

83. - Eiden-Offe nimmt den Begriff als theoretische Provokation ernst, um Brochs Problematisierung der Unterscheidbarkeit von Demokratie und Totalitarismus vor dem Hintergrund aktueller Theoriedebatten zu erhellen. Vgl. Patrick Eiden-Offe, Das Reich der Demokratie. Hermann Brochs ,,Der Tod des Vergil“, München, Wilhelm Fink, 2011, S. 22.

84. - Wie Weidner anmerkt, ist die Wortwahl typisch für die manchmal unklaren Grenzen zwischen totalitärem und demokratischem Gedankengut der 1940er Jahre. Daniel Weidner, „,Fröhliche Apokalypse‘. Massenwahn und parabolisches Erzählen. Hermann Brochs Rückblick auf Europa“, in: Alfred Bodenheimer/Barbara Breysach (Hrsg.), Abschied von Europa. Jüdisches Schreiben zwischen 1930 und 1950, München, edition text \& kritik, 2011, S. 172-193, hier S. 181.

85. - H. Broch, Massenwahntheorie, a.a.O., S. 366.

86. - Ebd., S. 532.

87. - Ebd., S. 380.

88. - Eiden-Offe charakterisiert den „Heilsbringer“ als Figur des Aufschubs, die wie der „Führer“ auf Richtungsgebung zielt. Als Symbolisierung sei diese im Falle des Heilsbringers ethischer, im Falle des Demagogen ästhetischer Art. Vgl. P. Eiden-Offe, 
einen neuen Wertzusammenschluss will er dennoch nicht aufgeben. Allerdings wäre dieser sicherlich nicht durch eine romantische oder reaktionäre Imitation vergangener Kulturphasen zu realisieren, wie sie seiner Stiltheorie zufolge im Ornamentalen als reinem Dekor versinnbildlicht werden ${ }^{89}$. Broch konstruiert um den leeren Platz Gottes eine politische Theologie der Demokratie, wenn er von der Hoffnung auf ein neues Irdisch-Absolutes spricht. Dieses wird als das konstituiert, was „nicht sein darf": Konzentrationslager, Versklavung, Todesstrafe. Er formuliert das Irdisch-Absolute ex negativo und geht damit in diesem einen Punkt in eine ähnliche Richtung wie Adorno, wenn jener anmerkt, dass der Ort der Moralphilosophie „mehr in der konkreten Denunziation des Unmenschlichen als in der unverbindlichen und abstrakten Situierung des Seins des Menschen zu suchen ist" $" 90$.

Im Oktober 1945 schickt Broch einen Brief an Max Horkheimer. Er hatte das Manuskript der Philosophischen Fragmente von Horkheimer und Adorno gelesen, das zwei Jahre später als Dialektik der Aufklärung veröffentlicht wurde. In seinem Brief bestätigt er Horkheimers/Adornos These, wonach die ,(innerlogische) Entwicklung der Ratio an der Entwicklung des heutigen Weltzustand[es] mitschuldtragend ist, ja ihn eigentlich herbeiführte“91. Dies sei ihm bei seinen massenpsychologischen Untersuchungen deutlich geworden. Er kritisiert jedoch die negative Zukunftsprognose der Autoren: Die Philosophischen Fragmente, diese „Apokalypse der Ratio“, sei tatsächlich bereits ein Zeugnis von deren „Selbstbesinnungs-Kräfte[n]“. Und er fügt hinzu: „sie [die Menschheit] verfügt in jeder einzelnen Menschenseele, ja in der Ratio selber [über] einen unerschöpflichen Möglichkeits-Fond, [...] aus dem immer wieder das Wunder -- das rationale Wunder-- geschöpft wird“92. Adorno hält zwei Jahrzehnte später, im Jahr 1967, in Wien einen Vortrag über den zeitgenössischen Rechtradikalismus und bezeichnet hier die rechtsextremen Bewegungen als Narben der Demokratie: zu ihrer Bekämpfung setzt er auf die Entlarvung der Machttechniken durch genaue Beschreibung, um die projektiven Momente der rechtsradikalen Weltsicht aufzudecken. Es bleibe doch nur auf die ,durchschlagende Kraft der Vernunft“ zu setzen ${ }^{93}$.

Das Reich der Demokratie, a.a.O., S. 66.

89. - P. M. Lützeler, „Ornament“, a.a.O., S. 37.

90. - Theodor W. Adorno, Probleme mit der Moralphilosophie, Suhrkamp, Frankfurt a.M., 1996, S. 261. Vgl. Paul Michael Lützeler, „Zweifel als Grundimpuls der Moderne“, in: Ders., Hermann Broch und die Moderne, a.a.O., S. 103-123, hier S. 109.

91. - Hermann Broch an Max Horkheimer, 2.10.1945, in: Max Horkheimer, Briefwechsel 1941-1948, Gesammelte Schriften Bd. 17, hrsg. von Gunzelin Schmid Noerr, Frankfurt a.M., Fischer Verlag, 1996, S. 652-656, hier S. 653.

92. - Ebd., S. 654.

93. - Theodor W. Adorno, Aspekte des neuen Rechtsradikalismus. Ein Vortrag, mit einem Nachwort v. Volker Weiß, Berlin, Suhrkamp 2019, S. 55. 
Dies hieße aber nicht zu moralisieren, sondern reale Interessen anzusprechen, und schließlich: anstatt ethischer Appelle an die Humanität zu formulieren auf jene unheilvollen Konsequenzen des Rechtsradikalismus hinzuweisen, die dieser auch für seine Anhänger hat ${ }^{94}$. 
